\title{
Data Movement in Flash Memories
}

\author{
Anxiao (Andrew) Jiang \\ Computer Science Department \\ Texas A\&M University \\ College Station, TX 77843 \\ ajiang@cse.tamu.edu
}

\author{
Michael Langberg \\ Computer Science Division \\ Open University of Israel \\ Raanana 43107, Israel \\ mikel@openu.ac.il
}

\author{
Robert Mateescu \\ Electrical Engineering Dept. \\ Caltech \\ Pasadena, CA 91125 \\ mateescu@paradise.caltech.edu
}

\author{
Jehoshua Bruck \\ EE \& CNS Dept. \\ Caltech \\ Pasadena, CA 91125 \\ bruck@caltech.edu
}

\begin{abstract}
NAND flash memories are the most widely used non-volatile memories, and data movement is common in flash storage systems. We study data movement solutions that minimize the number of block erasures, which are very important for the efficiency and longevity of flash memories. To move data among $n$ blocks with the help of $\Delta$ auxiliary blocks, where every block contains $m$ pages, we present algorithms that use $\Theta\left(n \cdot \min \left\{m, \log _{\Delta} n\right\}\right)$ erasures without the tool of coding. We prove this is almost the best possible for non-coding solutions by presenting a nearly matching lower bound. Optimal data movement can be achieved using coding, where only $\Theta(n)$ erasures are needed. We present a coding-based algorithm, which has very low coding complexity, for optimal data movement. We further show the NP hardness of both coding-based and noncoding schemes when the objective is to optimize data movement on a per instance basis.
\end{abstract}

\section{INTRODUCTION}

NAND flash memories are the most widely used nonvolatile memories due to their high data density and efficiency. In a NAND flash memory, cells are organized as blocks. A block has about $10^{5}$ cells, and a cell can store one or more bits. Every block is partitioned into pages, where a page is the unit of a read or write operation. A prominent property of flash memories is block erasure. It means to change any stored data, the whole block must be erased first before rewriting. Block erasures significantly decrease the longevity and the speed of flash memories, so it is very important to reduce them [5].

Flash memories often store a large amount of data, and data movement is very useful for reassembling files, wear leveling, and in-place computation. We consider the basic form where the data in different pages need to be switched. This problem was first studied in [11], where coding-based data movement is shown to minimize the number of erasures. In this paper, we significantly extend the known results by rigorously proving the gain of coding, presenting efficient data movement algorithms, and showing the NP hardness of perinstance optimization.

In the data movement problem, there are $n$ blocks, where each block has $m$ pages of data. The $m n$ pages of data need to be moved into each other's positions as required. $\Delta$ empty auxiliary blocks can be used to help data movement. The objective is to minimize the number of block erasures in the data movement process. We present efficient algorithms that use $\Theta\left(n \cdot \min \left\{m, \log _{\Delta} n\right\}\right)$ erasures without the tool of coding. We prove it is nearly the best possible by proving a close lower bound. Since coding-based solutions require $\Theta(n)$ erasures, this result rigorously proves the benefit of coding.

We present a strictly optimal coding-based algorithm for $\Delta=1$ with at most $2 n-1$ erasures. It has very low coding complexity. We further show that if the objective is to optimize data movement on a per instance basis, the problem is NP hard for both coding and non-coding schemes. Nevertheless, the coding technique in the above algorithm can be readily utilized in any per-instance-optimal solution.

A number of recent works have studied coding for rewriting [4], [6], [8], [10], [13] and error correction [9], [12] in flash memories at the cell level. There are also many works studying algorithms and data structures for flash data-storage systems [5]. This paper focuses on coding for data movement at the page level, and the results can be used to design more efficient flash storage systems.

The rest of the paper is organized as follows. Section II defines the data movement problem. Section III presents data movement algorithms without coding, and section IV derives a corresponding lower bound. Section $\mathrm{V}$ presents an efficient coding scheme for optimal data movement. Section VI studies the complexity and approximation of per-instance optimization. Section VII presents the conclusions.

\section{NOTATIONS AND CONCEPTS}

There are $n$ blocks containing data, denoted by $B_{1}, \ldots, B_{n}$. Every block consists of $m$ pages. For $i=1, \ldots, n$, the pages in $B_{i}$ are called $p_{i, 1}, \ldots, p_{i, m}$. For $1 \leqslant i \leqslant n$ and $1 \leqslant j \leqslant m$, the data originally stored in $p_{i, j}$ is denoted by $d_{i, j}$. There is a function $\alpha$ :

$$
\alpha:\{1, \ldots, n\} \times\{1, \ldots, m\} \rightarrow\{1, \ldots, n\}
$$

such that $\forall 1 \leqslant i \leqslant n, \mid\{(a, b) \mid 1 \leqslant a \leqslant n, 1 \leqslant b \leqslant$ $m, \alpha(a, b)=i\} \mid=m$. Our objective is to move (i.e., write) the data $d_{i, j}$ into a page in block $B_{\alpha(i, j)}$, for $1 \leqslant i \leqslant n$ and $1 \leqslant j \leqslant m$. There are $\Delta$ empty extra blocks, called auxiliary blocks, that we can use to help move data. (Every auxiliary block also has $m$ pages.) For reliability, it is required that during the data-movement process, the data in these $n+\Delta$ blocks must always be sufficient for recovering all the original data. In the end, the auxiliary blocks should return to the empty state. We measure the cost of data movement by the total number of block erasures. (Note that changing any data in a block requires erasing the block first.) The solution that minimizes the number of erasures is called optimal.

There exist two types of solutions, i.e., solutions with and without coding. In a solution without coding, data are simply copied from page to page. In a coding-based solution, the data written into a page can be any function of the existing data.

Example 1. We show an example without coding where $m=3$, $n=4$ and $\Delta=2$. Each page is indexed by its label (the 
destination block), and its content (the card suit). For example the pages indexed by $1_{\triangleright}, 1_{\diamond}$ and $1_{\diamond}$ should be moved to the first block. In the first step, the content of the first block is copied to the auxiliary memory, and the first block is then erased. In the second step, the pages labeled with 1 are copied into the first block. In the third step, before erasing block 2, we copy only $3_{\diamond}$ and $4_{\diamond}$ to the auxiliary memory, because $1_{\diamond}$ already appears in block 1. After several steps, we realize the desired data movement.

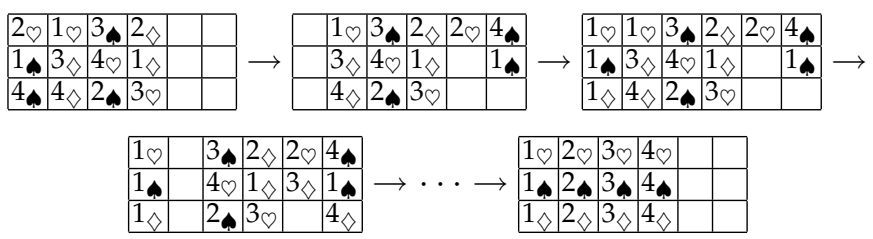

It is known that coding-based solutions can optimize data movement [11], which use at most $2 n-1$ erasures. We now rigorously prove the gain of coding, and show efficient datamovement algorithms.

\section{DATA MOVEMENT Without CODING}

In this section, we present two algorithms for data movement without coding. They use $n\left\lceil\log _{\Delta} n\right\rceil+\frac{3 n}{2}$ and $\frac{5 n m}{2}$ erasures, respectively. This shows that without coding, $\Theta(n$. $\left.\min \left\{m, \log _{\Delta} n\right\}\right)$ erasures are sufficient for data movement. Here we assume $\Delta \geqslant 2$, because when $\Delta=1$ there are cases that cannot be solved without using coding [11].

\section{A. Block-merging Algorithm}

Given a positive integer $i$, let $[i]$ denote $\{1,2, \ldots, i\}$. Call the $\Delta$ auxiliary blocks $B_{n+1}, \ldots, B_{n+\Delta}$. For $n+1 \leqslant i \leqslant$ $n+\Delta$, denote the $m$ pages in $B_{i}$ by $p_{i, 1}, \ldots, p_{i, m}$. In the data movement process, data are copied from page to page. At any given moment, if a page $p_{i_{1}, j_{1}}$ stores the data $d_{i_{2}, j_{2}}$, the we use $\phi_{i_{1}, j_{1}}$ to denote $\alpha\left(i_{2}, j_{2}\right)$. That is, the data in page $p_{i_{1}, j_{1}}$ need to be moved into block $B_{\phi_{i_{1}, j_{1}}}$ in the end.

Definition 2. Let $S \subseteq[n+\Delta]$. The blocks $\left\{B_{i} \mid i \in S\right\}$ are called "semi-sorted" if there exists a bijection function $\pi: S \rightarrow$ $S$ (that is, a permutation of the elements in $S$ ) such that $\forall i_{1} \neq$ $i_{2} \in S$ and $j_{1}, j_{2} \in[m]$, if $\pi\left(i_{1}\right)<\pi\left(i_{2}\right)$, then $\phi_{i_{1}, j_{1}} \leqslant \phi_{i_{2}, j_{2}}$.

Let's use the help of semi-sorted blocks to move data. For simplicity, in the following we assume $n$ is a power of $\Delta$, namely, $n=\Delta^{z}$ for some integer $z$. We will extend the results for general $n$ later. As the beginning step, we partition $[n]$ into $n / \Delta$ subsets $S_{1}, \cdots, S_{n / \Delta}$, where $\left|S_{i}\right|=\Delta$ for $1 \leqslant i \leqslant$ $n / \Delta$. We create $n / \Delta$ sets of semi-sorted blocks as follows. We first copy the data in $\left\{B_{i} \mid i \in S_{1}\right\}$ into the $\Delta$ auxiliary blocks $\left\{B_{i} \mid n+1 \leqslant i \leqslant n+\Delta\right\}$ such that the blocks $\left\{B_{i} \mid n+\right.$ $1 \leqslant i \leqslant n+\Delta\}$ become semi-sorted blocks. Then for $i=$ $2, \ldots, n / \Delta$, we erase the blocks $\left\{B_{j} \mid j \in S_{i-1}\right\}$, and copy the data in $\left\{B_{j} \mid j \in S_{i}\right\}$ into $\left\{B_{j} \mid j \in S_{i-1}\right\}$ such that the blocks $\left\{B_{j} \mid j \in S_{i-1}\right\}$ become semi-sorted. Finally we erase the blocks $\left\{B_{i} \mid i \in S_{n / \Delta}\right\}$. This way, we have created $n / \Delta$ sets of semi-sorted blocks using $n$ erasures.

Clearly, our final objective is to make $\left\{B_{1}, \cdots, B_{n}\right\}$ one big set of semi-sorted blocks. How can we combine the $n / \Delta$ smaller sets of semi-sorted blocks we already have to achieve this objective? Let's use $T_{1}, \ldots, T_{n / \Delta}$ to denote our $n / \Delta$ sets of semi-sorted blocks, and use $T_{0}$ to denote the set of $\Delta$ empty blocks. We first show how to combine $T_{1}, \cdots, T_{\Delta}$ into one bigger set of semi-sorted blocks. We copy data from $T_{1}, \ldots, T_{\Delta}$ into the empty blocks (one empty block at a time) with the following rule: "for two pages $p_{i_{1}, j_{1}}$ and $p_{i_{2}, j_{2}}$ of $T_{1}, \ldots, T_{\Delta}$, if $\phi_{i_{1}, j_{1}}<\phi_{i_{2}, j_{2}}$, then the data in page $p_{i_{1}, j_{1}}$ is copied before the data in $p_{i_{2}, j_{2}}$." (Note that for every $T_{i}$ $(1 \leqslant i \leqslant \Delta)$, the data in it are already sorted, so they just need to be copied sequentially block after block.) For a block in $T_{i}(1 \leqslant i \leqslant \Delta)$, once its data are all copied, we erase it so that it becomes empty and can have data moved into it later. Can we keep moving data this way so that in the end, the data in $T_{1}, \cdots, T_{\Delta}$ are moved into $\Delta^{2}$ blocks, which are semisorted? The answer is yes, because in the above procedure there is always place to move data into: Every time we have completely filled a number of empty blocks, if we look at the blocks in $T_{1}, \ldots, T_{\Delta}$ whose data have been partially copied, their un-copied data together can fill at most $\Delta-1$ empty blocks. Since we have $\Delta$ empty blocks to begin with, there is always an empty block to copy data into.

Using the same method, we can combine the $n / \Delta$ sets of semi-sorted blocks into $n / \Delta^{2}$ bigger sets of semi-sorted blocks. By repeatedly using this approach $\log _{\Delta} n$ times, we can get $n$ blocks that are semi-sorted. All left to do is to move the data into their final positions, which takes at most $3 n / 2$ erasures. In total, this algorithm uses at most $n \log _{\Delta} n+\frac{3 n}{2}$ erasures. For general values of $n$, this algorithm uses at most $n\left\lceil\log _{\Delta} n\right\rceil+\frac{3 n}{2}$ erasures.

\section{B. Algorithm based on Block-permutation Sets}

We now present an algorithm that uses $O(n m)$ erasures.

Definition 3. A set of $n$ pages $p_{1, j_{1}}, p_{2, j_{2}}, \ldots, p_{n, j_{n}}$ is a "blockpermutation set" if $\left\{\alpha\left(1, j_{1}\right), \alpha\left(2, j_{2}\right), \ldots, \alpha\left(n, j_{n}\right)\right\}=[n]$.

It is known that the $n m$ pages in $B_{1}, \ldots, B_{n}$ can be partitioned into exactly $m$ block-permutation sets [11]. Without loss of generality (w.l.o.g.), let's assume that for $i=1, \ldots, m$, the $n$ pages $p_{1, i}, p_{2, i}, \ldots, p_{n, i}$ form a block-permutation set. (Since a block contributes exactly one page to every blockpermutation set, this is just a matter of labelling.) By definition, the data of the $n$ pages in a block-permutation set need to be permuted in the $n$ blocks $B_{1}, \ldots, B_{n}$. In the following, we use this property to move data, using only two auxiliary blocks (which we will call $B_{n+1}$ and $B_{n+2}$ ).

Consider a block-permutation set $p_{1, j}, p_{2, j} \ldots, p_{n, j}$. Since a permutation consists of "permutation cycles", let's consider such a cycle of length $z \leqslant n: p_{i_{0}, j}, p_{i_{1}, j}, \ldots, p_{i_{z-1}, j}$. That is, for $k=0,1, \ldots, z-1, \alpha\left(i_{k}, j\right)=i_{(k+1)} \bmod z$. Roughly speaking, with the two auxiliary blocks, we can move the data inside the cycle to their right places without moving any data outside the cycle. The basic idea is that with one auxiliary block, we can cyclically shift the data inside the cycle. With the other auxiliary block, we can use it to temporarily hold the data outside the cycle when the corresponding block is erased. Due to space limitations, we omit a more detailed description 
of our procedure. Such a description appears in the full version of our work [7].

With the outlined procedure, we can move $z$ pages of data in a cycle using $2 z+1$ erasures. In the same way, we can move the $n$ pages of data in a block-permutation set using at most $5 n / 2$ erasures. Furthermore, we can move all the $n m$ pages using $5 \mathrm{~nm} / 2$ erasures.

We have designed another efficient data-movement algorithm, called the bit-fixing algorithm, that allows straightforward implementation in flash memories. We present it in the appendix. All in all, we conclude:

Theorem 4. Let $\Delta \geqslant 2$. When coding is not used, the data movement problem can be solved using at most

$$
\min \left\{n\left\lceil\log _{\Delta} n\right\rceil+\frac{3 n}{2}, \frac{5 n m}{2}\right\}=\Theta\left(n \cdot \min \left\{m, \log _{\Delta} n\right\}\right)
$$

erasures.

\section{A LOWER BOUND}

In this section, we prove that without coding, a data-movement algorithm needs $\Omega(n$. $\left.\min \left\{m,\left(\log _{\Delta} n\right) /\left(\log _{\Delta}^{*} n\right)\right\}\right)$ erasures. ${ }^{1}$ Since $\log _{\Delta}^{*} n$ is practically a very small number, this lower bound is very close to the upper bound shown in Theorem 4.

\section{A. Model}

In this section, to simplify our notation, we use an equivalent model defined as follows: Our data is modeled as an $m \times(n+\Delta)$ matrix $A=\left(a_{i, j}\right)_{m \times(n+\Delta)}$. Initially, the data in the sub matrix consisting of the first $n$ columns contains all the elements in the set $[\mathrm{mn}]$; and the remaining (auxiliary) entries are empty. Namely, each of the $m n$ entries in the first $n$ columns have distinct values, and all $x \in[m n]$ appear in these columns. The value $x$ of $a_{i, j}$ indicates that the data in page $p_{i, j}$ needs to be moved to location $i^{\prime}, j^{\prime}$ where $x=\left(i^{\prime}-1\right)+\left(j^{\prime}-1\right) * m\left(\right.$ namely, $i^{\prime}-1=(x \bmod m)$ and $\left.j^{\prime}-1=\lfloor x / m\rfloor\right)$. In other words, after the data movement, the final matrix $A^{*}$ will include the elements of $[\mathrm{mn}]$ in the first $n$ columns in increasing order; the remaining $\Delta$ columns will be empty. We use $B_{i}$ to denote the set of values that appear in the $i$-th column of $A\left(B_{i} \subset[m n]\right)$. We assume throughout that $\Delta \leqslant n$ (otherwise the problems studied here become trivial).

In general, our objective is to perform operations to the initial matrix $A$, such that the data in each page is moved to its corresponding location. We allow two types of operations: (a) Copying data from a current page to an empty page. This corresponds to writing the value of an entry $a_{i j}$ into a location that was previously empty. Copying data is thought of as a costless operation. (b) Erasing a column of data. This corresponds to erasing the value of all entries in a given column. After the operation, all entries in the given column will be empty. Erasing data is thought of as a costly operation.

\footnotetext{
${ }^{1}$ Here $\log _{\Delta}^{*} n$ is the iterated logrithm of $n$, which is defined as the number of times the logrithm function must be iteratively applied before the result is less than or equal to 1 . Namely, $\log _{\Delta}^{*} n=1+\log _{\Delta}^{*}\left(\log _{\Delta} n\right)$ for $n>\Delta$. Notice that $\log _{\Delta}^{*} n$ grows very slowly with $n$.
}

Given an initial matrix, using the two operations above, our objective is to reach the final matrix $A^{*}$ specified above. In this section, we present lower bounds to the number of erasures needed to reach this goal.

\section{B. Proof outline}

To prove our lower bounds, we consider the directed configuration graph $G=(S, E)$ of our process. The vertex set $S$ of our graph will include all possible matrices $A$ that can be reached by performing operations (a) and (b) above on the initial matrix $A$. To be precise, to allow a clean analysis, we will restrict ourselves to the set of matrices $A^{\prime}$, reachable from $A$, that have exactly $\Delta m$ empty entries; and that the remaining $m n$ entries include the set $[m n]$ (one entry per element). It is not hard to verify that this is without loss of generality. On one hand for every $x \in[m n]$ our intermediate matrix $A^{\prime}$ must include an entry of value $x$, otherwise we will not be able to reach the final matrix $A^{*}$. On the other, we may assume that there are exactly $\Delta m$ empty entries as any value $x \in[m n]$ that appears in two positions is redundant. We define a slightly different operation on our matrix that preserves the above restrictions and combines operations (a) and (b) above. (ab) Erase and copy: Erase a column of data, and copy its content onto the empty locations in the matrix (these empty locations may include the currently erased column). Our objective is, starting from our initial matrix $A$, to perform a minimum number of $(\mathrm{ab})$ operations to reach $A^{*}$.

We have yet to define the edge set $E$ of our graph $G$. A pair $\left(A^{\prime}, A^{\prime \prime}\right)$ is a directed edge in $E$ if using a single (ab) operation one can transform $A^{\prime}$ into $A^{\prime \prime}$. The distance between a matrix $A$ and $A^{*}$ is the shortest path in $G$ between $A$ and $A^{*}$, and corresponds to the minimum number of (ab) operations needed to transform $A$ into the desired matrix $A^{*}$. We will show the existence of an initial matrix $A$ for which this distance is at least a certain lower bound $l b$.

Our proof has two steps. First, we show that the diameter of $G$ is at least $l b$ (namely there exist two matrices $A^{\prime}$ and $A^{\prime \prime}$ which are far apart.

Theorem 5. The diameter of $G$ is at least $l b=\Theta(n$. $\left.\min \left(m, \log _{\Delta} n / \log _{\Delta}^{*} n\right)\right)$.

We then show that this suffices to prove our assertion.

Corollary 6. There exists a matrix $A$ such that the distance between $A$ and $A^{*}$ is at least $\Theta\left(n \cdot \min \left(m, \log _{\Delta} n / \log _{\Delta}^{*} n\right)\right)$.

The proof of Corollary 6 is fairly simple and is omitted from this extended abstract. A detailed proof can be found in the full version of the paper [7].

1) Outline for proof of Theorem 5: To prove Theorem 5 (our lower bound on the diameter of $G$ ) we use a variation of the following naive idea. For a matrix $A$, let $d_{A}$ be the out-degree of $A$ in $G$. Assume one could prove that $d_{A}$ is bounded by $D$ for all matrices $A \in S$. This implies that a walk of length $\ell$ in $G$ starting from $A$ can reach at most $D^{\ell}$ different matrices of $S$. One can now deduce that the diameter of $G$ is at least the smallest $\ell$ such that $D^{\ell} \geqslant|S|$; or in other words $l b \geqslant \log _{D}|S|$. 
Applying this proof technique "as is" on our graph $G$ will not yield a lower bound greater than $n$. We thus consider two modifications. First of all, we consider a slightly different graph $G^{\prime}=\left(S^{\prime}, E^{\prime}\right)$, which is a homomorphic image of $G$. Namely, for a given matrix $A \in S$, let $B_{i}$ be the set of values that appear in the $i$ 'th column of $A$. We will identify $A$ with the tuple $B_{A}=\left(B_{1}, \ldots, B_{n+\Delta}\right)$; and $S^{\prime}=\left\{B_{A} \mid\right.$ $A \in S\}$ will consist of the set of such tuples. Two tuples $B=\left(B_{1}, \ldots, B_{n+\Delta}\right)$ and $B^{\prime}=\left(B_{1}^{\prime}, \ldots, B_{n+\Delta}^{\prime}\right)$ are connected by a directed edge in $E^{\prime}$ iff there exists matrices $A$ and $A^{\prime}$ with corresponding tuples $B$ and $B^{\prime}$ that are connected in $G$. It is not hard to verify that the diameter of $G$ is no less than the diameter of $G^{\prime}$. (In fact, we can also show that the diameter of $G$ is no more than the diameter of $G^{\prime}$ plus $n+\Delta$. So the two diameters are the same up to an additive factor of $n+\Delta$.) We conclude that a lower bound of $\Theta\left(n \cdot \min \left(m, \log _{\Delta} n / \log _{\Delta}^{*} n\right)\right)$ for the diameter of $G^{\prime}$ will imply the same lower bound for $G$.

Up to this point we have discussed the first variation of the naive idea presented above: replacing the graph $G$ by $G^{\prime}$. However, bounding the degree $D^{\prime}$ of $G^{\prime}$ will not suffice to obtain a lower bound of $\log _{D^{\prime}}\left|S^{\prime}\right|$ larger than $n$. The main reason is that the maximum degree $D^{\prime}$ of $G^{\prime}$ is too large. But we have noticed that only rarely may one visit vertices of $G^{\prime}$ with large degree approaching this bound. Typically the degree of the vertices at hand will be fairly small. To utilize this observation, we consider the maximum number of vertices in $S^{\prime}$ reachable from a given vertex $B$ by $\alpha>1$ steps instead of a single step (for a single step, this value is exactly the vertex out-degree). This corresponds to the study of the $\alpha$ transitive closure of $G^{\prime}$ sometimes denoted as $\left(G^{\prime}\right)^{\alpha}$. Namely, $\left(G^{\prime}\right)^{\alpha}$ consists of the vertex set $S^{\prime}$, where two vertices are connected by an edge iff there is a path of length at most $\alpha$ between them in $G^{\prime}$. Clearly, if $\left(G^{\prime}\right)^{\alpha}$ has diameter diam, then $G^{\prime}$ has diameter at least diam $\cdot \alpha$. Studying $\left(G^{\prime}\right)^{\alpha}$ instead of $G^{\prime}$ allows us to average out the differences between the degree of vertices in $G^{\prime}$ and to obtain the desired bound. In what follows we analyze the size of $S^{\prime}$ and the value $D e g_{\alpha}$ of the maximum out-degree in $\left(G^{\prime}\right)^{\alpha}$. We then deduce a bound on the diameter of $G^{\prime}$ of $l b \geqslant \Theta\left(\alpha \log _{\operatorname{Deg}_{\alpha}}\left|S^{\prime}\right|\right)$ which in turn implies the assertion of Theorem 5. In what follows, for two functions $f$ and $g$, the notation $f \simeq g$ will represent the fact that $\log f=\Theta(\log g)$. We also assume that $\Delta<n$ (otherwise there is a trivial lower and upper bound of $n$ ).

\section{The state space $S^{\prime}$}

We start by bounding (from below) the total number of configurations in the state space $S^{\prime}$ at hand.

$$
\left|S^{\prime}\right| \geqslant\left(\begin{array}{c}
n m+\Delta m \\
\Delta m
\end{array}\right) \frac{(n m) !}{(m !)^{n}} \geqslant n^{n m}
$$

We explain our bound: We first choose the $\Delta m$ location for the empty entries of our data matrix. This determines the size of the sets $B_{1}, \ldots, B_{n+\Delta}$, say $m_{1}, \ldots, m_{n+\Delta}$ where each $m_{i}$ is at most $m$. We then decide on the content of each set $B_{i}$. If the sets were ordered, the number of configurations would be exactly $(\mathrm{nm})$ !. As they are not ordered this number should be divided by $\prod_{i=1}^{n+\Delta}\left(m_{i}\right)$ !. It is not hard to verify that $\Pi_{i=1}^{n+\Delta}\left(m_{i}\right) ! \leqslant(m !)^{n}$ as $\sum_{i=1}^{n+\Delta} m_{i}=n m$ and each $m_{i}$ is at most $m$.

\section{Deg $g_{\alpha}$ : number of vertices reachable in $\alpha$ steps}

We now bound (from above) the number of vertices $D e g_{\alpha}$ that can be reached using $\alpha$ steps from any given initial vertex in $S^{\prime}$. We take $n>\alpha>\Delta$. We define $D(\alpha)$ to be the number of different configurations a certain set of $\alpha$ columns can take in $\alpha$ steps.

$$
D e g_{\alpha} \leqslant\left(\begin{array}{c}
n+\Delta \\
\alpha
\end{array}\right)\left(\begin{array}{c}
\alpha m \\
\Delta m
\end{array}\right)(n+\Delta)^{\Delta m} D(\alpha) \simeq n^{\alpha+\Delta m} D(\alpha)
$$

We explain our bound: We first pick $\alpha$ columns out of the $n+\Delta$ columns. There are now two types of changes that may have been made in the data matrix, changes inside the $\alpha$ columns we picked (referred to as internal columns) and changes outside these columns (referred to as external columns). We start with external changes. The external empty spaces may have been filled with elements from the internal columns. This can be bounded by $\left(\begin{array}{c}\alpha m \\ \Delta m\end{array}\right)$ to choose the internal elements, and $(n+\Delta)^{\Delta m}$ to distribute them among the external empty spaces (notice that there are at most $\Delta m$ such empty spaces). Now we are left to consider the number of internal configurations one may obtain. We denote this value by $D(\alpha)$. Namely, $D(\alpha)$ equals the number of possible configurations obtainable in a given set of $\alpha$ columns when erasing these columns one after the other. It is clear that $D(\alpha) \leqslant \alpha^{m \alpha}$, Indeed, for a rough bound notice that internal entries must appear in one of the $\alpha$ columns. Plugging in this value of $D(\alpha)$ will already yield nice results. However, to tighten the results we compute $D(\alpha)$ recursively. Namely, for $\alpha>\beta>\Delta$ it holds that

$$
D(\alpha) \leqslant\left[\left(\begin{array}{c}
\alpha \\
\beta
\end{array}\right)\left(\begin{array}{c}
\beta m \\
\Delta m
\end{array}\right) \alpha^{\Delta m} D(\beta)\right]^{\frac{\alpha}{\beta}} \simeq\left[\alpha^{\beta+\Delta m} D(\beta)\right]^{\frac{\alpha}{\beta}}
$$

Again, we explain our bound. We would like to express the number of internal configuration obtainable in $\alpha$ steps $D(\alpha)$ by the number of internal configurations obtainable in $\beta$ steps $D(\beta)$ for $\beta$ smaller than $\alpha$. The analysis is similar to the previous one for $D e g_{\alpha}$. We first compute how many configurations can be obtained in $\beta$ steps, and then raise this number by $\alpha / \beta$. We start by picking $\beta$ columns out of the $\alpha$ at hand. For the upcoming discussion, we refer to the $\alpha$ columns as external columns and to the $\beta$ columns as internal columns. Again, there may be two types of changes in the configuration, external and internal. For external changes, we may fill some of the empty entries of the external columns with internal entries. This is counted for by $\left(\begin{array}{c}\alpha \\ \beta\end{array}\right)\left(\begin{array}{c}\beta m \\ \Delta m\end{array}\right) \alpha^{\Delta m}$. As before, the internal changes are attributed to $D(\beta)$.

We now compute our lower bound. We have the freedom to fix the values of $\alpha$ and $\beta$. For the first level of recursion, we fix $\alpha=\Delta \log _{\Delta} n$ and $\beta=\Delta \log _{\Delta} \log _{\Delta} n=\Delta \log _{\Delta}^{(2)} n$. It holds that $n>\alpha>\beta$. Thus, $D e g_{\alpha} \simeq n^{\alpha+\Delta m} D(\alpha)$ is approximately

$$
n^{\Delta \log _{\Delta} n+\Delta m}\left[\left(\Delta \log _{\Delta} n\right)^{\Delta \log _{\Delta}^{(2)} n+\Delta m} D\left(\Delta \log _{\Delta}^{(2)} n\right)\right]^{\frac{\log _{\Delta} n}{\log _{\Delta}^{(2)} n}}
$$


For $\Delta<\log _{\Delta} n$ it holds that

$$
n^{\frac{\log _{\Delta}^{(2)} n}{\log _{\Delta} n}} \simeq \Delta \log _{\Delta} n
$$

Thus we have that $D e g_{\alpha} \simeq$

$$
n^{\Delta \log _{\Delta} n+\Delta \log _{\Delta}^{(2)} n} n^{2 \Delta m} D\left(\Delta \log _{\Delta}^{(2)} n\right)^{\frac{\log _{\Delta} n}{\log _{\Delta}^{(2)} n}}
$$

For the second step of our recursion, we need to compute $D\left(\alpha^{\prime}\right)=D\left(\Delta \log _{\Delta}^{(2)} n\right)$. We do this by fixing $\alpha^{\prime}=\Delta \log _{\Delta}^{(2)} n$ and $\beta^{\prime}=\Delta \log _{\Delta}^{(3)} n$. For $\Delta<\log _{\Delta}^{(2)} n$ it holds that

$$
n^{\frac{\log _{\Delta}^{(3)} n}{\log _{\Delta}^{n}}} \simeq \Delta \log _{\Delta}^{(2)} n
$$

Thus we have that $D e g_{\alpha} \simeq$

$$
n^{\sum_{i=1}^{3}\left(\Delta \log _{\Delta}^{(i)} n+\Delta m\right)} D\left(\Delta \log _{\Delta}^{(3)} n\right)^{\frac{\log _{\Delta} n}{\log _{\Delta}^{(3)} n}}
$$

In general, we can continue the recursion as long as $\Delta<$ $\log _{\Delta}^{(i)} n$, while for the base we take $D(\Delta)=\Delta^{m \Delta}$. So all in all we get

$\operatorname{Deg}_{\alpha} \leqslant n^{\sum_{i=1}^{\log _{\Delta}^{*} n}\left(\Delta \log _{\Delta}^{(i)} n+\Delta m\right)} \Delta^{m \Delta \frac{\log _{\Delta} n}{\Delta}} \simeq n^{\Delta m \log _{\Delta}^{*} n} n^{\Delta \log _{\Delta} n}$

Finally, we evaluate our lower bound $l b \geqslant \alpha \frac{\log _{\Delta}\left|S^{\prime}\right|}{\log _{\Delta} D e g_{\alpha}} \simeq$ $\frac{\Delta m n \log _{\Delta} n}{\Delta m \log _{\Delta}^{*} n+\Delta \log _{\Delta} n}=\frac{n}{\log _{\Delta}^{*} n / \log _{\Delta} n+1 / m}$, which implies a lower bound of

$$
l b=\Theta\left(n \cdot \min \left(m, \log _{\Delta} n / \log _{\Delta}^{*} n\right)\right)
$$

Almost matching our upper bound of $\Theta\left(n \min \left(m, \log _{\Delta} n\right)\right)$.

\section{EFFicient Coding-BASEd Data Movement}

We now focus our attention on data movement using coding, where $\Delta=1$. It is known that with coding, $2 n-1$ erasures are sufficient and necessary in the worst case. What about minimizing the number of erasures for each given instance, instead of just the worst case? (Certainly, for some instances, fewer than $2 n-1$ erasures are needed.) Our coding-based solution will use the concept of "canonical labelling."

Definition 7. [11] Let $y \in[n-2]$. When we relabel the $n$ blocks $B_{1}, \ldots, B_{n}$ as $B_{1}^{\prime}, \ldots, B_{n}^{\prime}$, it is called a "canonical labelling with parameter $y$ " if for any $y+1 \leqslant i \leqslant n-2$ and $i+2 \leqslant j \leqslant n$, no data in $B_{j}^{\prime}$ need to be moved into $B_{i}^{\prime}$.

The following observation has been made in [11]: "Given an instance of the data-movement problem with $\Delta=1$, there exists a coding-based solution using $n+y+1$ erasures if and only if there exists a canonical labelling with parameters $y$." It is NP hard to find a canonical labelling with $y$ minimized [11]. However, we should notice that when $y=n-2$, any labelling is a canonical labelling, and that would give us the $n+y+1=$ $2 n-1$ erasures, which is worst-case optimal.

We present a very efficient coding-based algorithm that uses $n+y+1$ erasures once a canonical labelling with parameter $y$ is given. In the algorithm, a very small Galois field is used for computation ( $G F(q)$ with $q \geqslant 3)$, and it generates parity-check
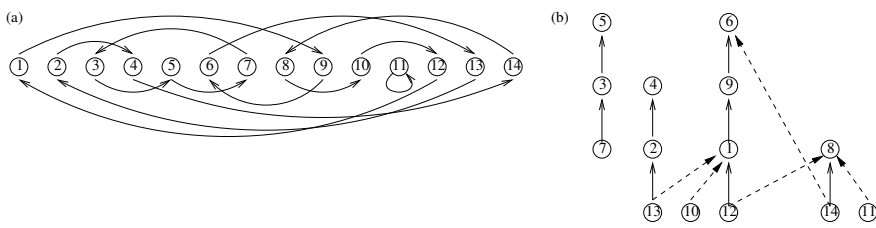

Figure 1. Example of the data-movement problem with $n=14$. We let $y=8$. (a) The data-movement graph $G_{d}$. If the data $d_{i}$ needs to be moved to page $p_{j}$, there is an edge from vertex $i$ to vertex $j$. (b) The symbol graph $G_{s}$. The solid edges are the black edges, and the dashed edges are the red edges.

symbols with a small number of additions on average. Before this work, it was unknown how to find such strictly-optimal and efficient solutions over small Galois fields [11]. Our algorithm achieves low coding complexity while minimizing the number of erasures.

The algorithm takes a canonical labelling with parameter $y$ as input. For simplicity, let's say that the original labelling $B_{1}, B_{2}, \ldots, B_{n}-$ is the canonical labelling. (It is just a matter of naming.) It has been shown in Section III that the $n m$ pages of $B_{1}, \ldots, B_{n}$ can be partitioned into $m$ block-permutation sets. Our algorithm will work the same way for the $m$ blockpermutation sets. So for simplicity, in the following, we will consider only one block-permutation set, and call its pages $p_{1}, p_{2}, \ldots, p_{n}$. For $i \in[n], p_{i}$ is the page in block $B_{i}$ and originally holds the data $d_{i}$. For $i \in[n]$, the function $\alpha(i)=j$ means that the data $d_{i}$ need to be moved into the page $p_{j}$. (Here $j \in[n]$.) By the definition of block-permutation set, we know that $\{\alpha(1), \ldots, \alpha(n)\}=\{1, \ldots, n\}$. If $\alpha(i)=j$, we say $\alpha^{-1}(j)=i$. By the definition of canonical labelling with parameter $y$, we know that for $i \in\{y+1, y+2, \ldots, n-2\}$, $\alpha^{-1}(i) \leqslant i+1$.

We will use only one auxiliary block. We call it $B_{0}$, and call its page $p_{0}$. In our algorithm, $B_{1}, \ldots, B_{y}$ will be erased twice, while $B_{0}$ and $B_{y+1}, \ldots, B_{n}$ will be erased only once.

We define a function $\beta(i)$ for $i \in\{y+2, y+3, \ldots, n\}$ :

- If $\alpha^{-1}(i-1) \leqslant y+1$ and $\alpha^{-1}(i-1) \neq \alpha(i)$, then $\beta(i)=\alpha^{-1}(i-1)$.

- If $y+2 \leqslant \alpha^{-1}(i-1) \leqslant i-1$, then $\beta(i)=\beta\left(\alpha^{-1}(i-\right.$ 1)).

- If $\alpha^{-1}(i-1)=i$, or if $\alpha^{-1}(i-1) \leqslant y+1$ and $\alpha^{-1}(i-$ 1) $=\alpha(i)$, then $\beta(i)=N U L L$.

We define the set $\gamma_{i}$, for $i \in[y+1]$, as follows:

$$
\gamma_{i}=\{j \mid \beta(j)=i, y+2 \leqslant j \leqslant n\} .
$$

Example 8. An example is shown in Fig. 1 (a). Here $n=14$ and $y=8$. For $i \in[n]$, if $\alpha(i)=j$ (that is, the data $d_{i}$ need to be moved into page $p_{j}$ ), we draw an edge from vertex $i$ to vertex $j$. We call the graph the data-movement graph $G_{d}$.

Here we have $\alpha(1)=9, \alpha(2)=4, \ldots, \alpha(14)=8$, and $\alpha^{-1}(1)=12, \alpha^{-1}(2)=13, \ldots, \alpha^{-1}(14)=4$. We also have $\beta(10)=1, \beta(11)=8, \beta(12)=8, \beta(13)=1, \beta(14)=6$. Correspondingly, $\gamma_{1}=\{10,13\}, \gamma_{6}=\{14\}, \gamma_{8}=\{11,12\}$. When $i \in[y+1]$ and $i \neq 1,6,8, \gamma_{i}=\varnothing$.

It is well known that a permutation consists of cycles.

Definition 9. A set of pages $p_{i_{0}}, p_{i_{1}}, \ldots, p_{i_{x-1}}$ is called a permutation cycle if for $j=0,1, \ldots, x-1, \alpha\left(i_{j}\right)=i_{j+1} \bmod x$. 
Here $i_{j} \in[n]$ for all $j$. Among the $x$ pages, $p_{\max \left\{i_{j} \mid 0 \leqslant j \leqslant x-1\right\}}$ is called the tail of the permutation cycle.

Example 10. The data-movement problem shown in Fig. 1 (a) has three permutation cycles: (1) $p_{1}, p_{9}, p_{6}, p_{13}, p_{2}, p_{4}, p_{14}$, $p_{8}, p_{10}, p_{12}$; (2) $p_{3}, p_{5}, p_{7}$; (3) $p_{11}$. Their tails are $p_{14}, p_{7}$ and $p_{11}$, respectively.

Let us build a directed graph $G_{s}$, called the symbol graph, as follows. $G_{s}$ has $n$ vertices labelled by $1, \ldots, n$, which correspond to the $n$ pages $p_{1}, \ldots, p_{n}$. The edges of $G_{s}$ have two colors: black and red. There is a black edge from vertex $i$ to vertex $j$ if $\alpha(i)=j, j \in[y+1]$ and $p_{j}$ is not the tail of its permutation cycle. There is a red edge from vertex $i$ to vertex $j$ if $y+2 \leqslant i \leqslant n, j \in[y+1]$ and $i \in \gamma_{j}$.

Example 11. An example of the symbol graph $G_{S}$ is shown in Fig. 1 (b). It corresponds to the data movement problem in Fig. 1 (a). The black edges of $G_{s}$ always form disjoint paths. In this example, the paths are: (1) $7 \rightarrow 3 \rightarrow 5$; (2) $13 \rightarrow 2 \rightarrow 4$; (3) 10 ; (4) $12 \rightarrow 1 \rightarrow 9 \rightarrow 6$; (5) $14 \rightarrow 8$; (6) 11 . We call them black paths. The "sources" of the above six black paths are vertices $7,13,10,12,14,11$, respectively, and their "sinks" are vertices 5, 4,10,6,8,11, respectively.

It is easy to see that a black path can have at most one vertex from $\{y+2, y+3, \ldots, n\}$; and if it does, that vertex must be its source. For a red edge, its beginning point must be the source of a black path. The end point of a red edge is either the end of a black path or the vertex $\alpha^{-1}(y+1)$.

We show that the symbol graph $G_{S}$ has a simple structure. Let $\bar{G}_{s}$ be the undirected version of $G_{s}$. That is, if we covert all the edges of $G_{s}$ to be undirected edges, we get $\bar{G}_{s}$.

Lemma 12. Every connected component of $\bar{G}_{s}$ has at most one cycle.

Proof: Let us remove all the vertices of $\bar{G}_{s}$ that are not in any cycle, and call the remaining graph $G_{0}$. Every cycle in $G_{0}$ must have both black and red edges. Note that the black edges belong to the disjoint black paths, and the red edges have the properties described in Example 11. Let $x$ denote the number of red edges in $G_{0}$. The beginning point of the $x$ red edges (if we view them as directed edges) belong to $x$ different black paths. Since every vertex in $G_{0}$ is in a cycle, and every beginning point of a red edge (if we view it as a directed edge) of $G_{0}$ is incident to exactly one red edge and one black edge, each of those $x$ black paths must also contain exactly one end point of a red edge (if we view it as a directed edge). So every vertex in $G_{0}$ has degree two. So $G_{0}$ consists of vertex-disjoint cycles. So all the cycles in $\bar{G}_{S}$ are vertex-disjoint. If a path in $\bar{G}_{s}$ connects two cycles, the path would start with a red edge; but that red edge would have to share the starting point of another red edge - the latter edge is in a cycle - which would be impossible. So every connected component of $\bar{G}_{s}$ has at most one cycle.

We define a function $w(j)$ for $j \in\{y+2, y+3, \ldots, n\}$. Given a cycle $C$ in $\bar{G}_{s}$, let us call its vertices $i_{1}, i_{2}, \ldots, i_{x}$. Then for $j \in\left\{i_{1}, i_{2}, \ldots, i_{x}\right\} \cap\{y+2, y+3, \ldots, n\}$, let $w(j)=$ -1 if $j=\max \left\{\left\{i_{1}, i_{2}, \ldots, i_{x}\right\} \cap\{y+2, y+3, \ldots, n\}\right\}$, and $w(j)=1$ otherwise. For a vertex $j \in\{y+2, y+3, \ldots, n\}$ not in any cycle of $\bar{G}_{s}$, let $w(j)=1$.

We now present the coding-based algorithm. It uses $n+$ $y+1$ erasures to move data. For simplicity, we choose the computation to be over $G F(3)$, whose elements are $\{0,1,-1\}$. It is also feasible to use $G F(q)$ with $q>3$.

\section{Algorithm 13 Coding-Based Data Movement}

Step 1: For $i=1,2, \ldots, y+1$, do:

- If $p_{i}$ is not the tail of its permutation cycle, write the data $d_{i}-d_{\alpha^{-1}(i)}-\sum_{j \in \gamma(i)} w(j) d_{j}$ into the page $p_{i-1}$; otherwise, write the data $d_{i}-\sum_{j \in \gamma(i)} w(j) d_{j}$ into the page $p_{i-1}$.

- Erase the block $B_{i}$.

Step 2: For $i=y+1, y+2, \ldots, n-1$, write the data $d_{\alpha^{-1}(i)}$ into the page $p_{i}$, then erase the block $B_{i+1}$.

Step 3: Write the data $d_{\alpha^{-1}(n)}$ into the page $p_{n}$, then erase the block $B_{y}$.

Step 4: For $i=y, y-1, \ldots, 1$, write the data $d_{\alpha^{-1}(i)}$ into the page $p_{i}$, then erase the block $B_{i-1}$.

Example 14. Let the data movement problem be as shown in Fig. 1 (a), and we use Algorithm 13 to move data. The data stored in the pages $p_{0}, \ldots, p_{14}$ during the data movement process are shown in Fig. 2. Note that $p_{1}, \ldots, p_{y}$ are erased twice each, while $p_{y+1}, \ldots, p_{n}$ and $p_{0}$ are erased once each. The total number of erasures is $n+y+1$.

\begin{tabular}{|c|c|c|c|c|c|c|c|c|c|c|c|}
\hline pages & & $P_{0}$ & \multicolumn{2}{|c|}{$p_{1}$} & \multicolumn{2}{|c|}{$p_{2}$} & \multicolumn{2}{|c|}{$p_{3}$} & $p_{4}$ & \multicolumn{2}{|c|}{$p_{5}$} \\
\hline \multirow[t]{3}{*}{ data } & & & \multicolumn{2}{|c|}{$d_{1}$} & \multicolumn{2}{|c|}{$d_{2}$} & \multicolumn{2}{|c|}{$d_{3}$} & $d_{4}$ & \multicolumn{2}{|c|}{$d_{5}$} \\
\hline & \multicolumn{2}{|c|}{$\begin{array}{c}d_{1}-d_{12} \\
-d_{10}-d_{13}\end{array}$} & \multicolumn{2}{|c|}{$d_{2}-d_{13}$} & \multicolumn{2}{|c|}{$d_{3}-d_{7}$} & \multicolumn{2}{|c|}{$d_{4}-d_{2}$} & $d_{5}-d_{3}$ & \multicolumn{2}{|c|}{$\begin{array}{c}d_{6}-d_{9} \\
+d_{14}\end{array}$} \\
\hline & & & \multicolumn{2}{|c|}{$d_{12}$} & \multicolumn{2}{|c|}{$d_{13}$} & \multicolumn{2}{|c|}{$d_{7}$} & $d_{2}$ & \multicolumn{2}{|c|}{$d_{3}$} \\
\hline pages & $p_{6}$ & \multirow{2}{*}{\multicolumn{2}{|c|}{$\frac{p_{7}}{d_{7}}$}} & $p$ & & $p_{9}$ & $p_{10}$ & $p_{1}$ & $p_{12}$ & $p_{13}$ & $p_{14}$ \\
\hline \multirow[t]{3}{*}{ data } & $d_{6}$ & & & \multicolumn{2}{|c|}{$\frac{p_{8}}{d_{8}}$} & $d_{9}$ & $d_{10}$ & $d_{1}$ & $d_{12}$ & $d_{13}$ & $d_{14}$ \\
\hline & $d_{7}$ & \multicolumn{2}{|c|}{$\begin{array}{c}d_{8}-d_{14} \\
-d_{12}-d_{11}\end{array}$} & \multicolumn{2}{|c|}{$d_{9}-d_{1}$} & $d_{1}$ & $d_{8}$ & $d_{1}$ & $d_{10}$ & $d_{6}$ & $d_{4}$ \\
\hline & $d_{9}$ & \multicolumn{2}{|c|}{$d_{5}$} & \multicolumn{2}{|c|}{$d_{14}$} & & & & & & \\
\hline
\end{tabular}

Figure 2. Example of the coding-based data movement algorithm.

To prove that the algorithm is correct, the key is to prove that at any moment of the data-movement process, all the data can be recovered by decoding the data currently stored in the pages. For that, it is helpful to observe the one-to-one correspondence between the data written into the pages and the vertices/edges of the symbol graph $G_{s}$ : Every vertex $i \in[n]$ of $G_{S}$ corresponds to the data $d_{i}$, and the edges entering a vertex $i$ correspond to the data written into $p_{i-1}$ before $p_{i}$ is erased. (That is why $G_{s}$ is called the symbol graph.) For example, let us look at vertex 1 in Fig. 1 (b). It has three incoming edges, respectively from vertex 12,10 and 13 . Those incoming edges correspond to the symbol $d_{1}-d_{12}-d_{10}-d_{13}$. From Fig. 2, we see that was the symbol written into $p_{0}$ (before $p_{1}$ is erased by the algorithm for the first time). The vertex 1 itself corresponds to the symbol $d_{1}$, which was initially stored in $p_{1}$ and later written into $p_{9}$ (which are also shown in Fig. 2). Algorithm 13 always sequentially writes and erases symbol pairs sharing a common variable (i.e., $d_{i}$ for some $i$ ), which correspond to a vertex and its adjacent edge(s) in $G_{s}$. 
This makes the stored symbols always linearly independent. The detailed proof for Algorithm 13's correctness is presented in [7].

\section{Hardness of Optimizing Data Movement for EACH INSTANCE}

In the last section, we have presented a coding-based algorithm that moves data using $n+y+1 \leqslant 2 n-1$ erasures, once a canonical labelling of $B_{1}, \ldots, B_{n}$ with parameter $y \leqslant n-2$ is given. On the other hand, there exists a data-movement solution using $n+y+1$ erasures if and only if there exists a canonical labelling with parameter $y$. So the presented algorithm is strictly optimal. However, it is NP hard to find an optimal coding-based solution if the the canonical labelling with minimized parameter $y$ is not given first [11]. A natural question is: What is the complexity of finding the best solution without coding for each specific instance of the data-movement problem? (Again, this optimization is per-instance instead of for the worst case.) We study this topic in this section.

We will prove the NP hardness of non-coding solutions for a slightly generalized version of the data-movement problem. Let's allow some original data to be just erased, instead of moved. More specifically, we change the function $\alpha$ to $\alpha:\{1, \ldots, n\} \times\{1, \ldots, m\} \rightarrow\{1, \ldots, n\} \cup\{\perp\}$, where $\alpha(i, j)=\perp$ means that the data $d_{i, j}$ just need to be erased, instead of moved. This is a very practical generalization, because in flash memories, there are usually pages whose data are no longer useful, and such pages are labelled as "invalid" in flash memories to be erased later [5]. Let us call this version the generalized data-movement problem.

For $i, j \in[n]$, let $d(i \rightarrow j)$ denote the number of pages of data that need to move from block $B_{i}$ to block $B_{j}$. That is, $d(i \rightarrow j)=|\{k \mid k \in[m], \alpha(i, k)=j\}|$. We now define a concept called the movement graph $G_{m}$.

Definition 15. (MOVEMENT GRAPH) Corresponding to a generalized data-movement problem, we build a directed graph $G_{m}=(V, E)$ as follows. We let $V=\left\{v_{1}, v_{2}, \cdots, v_{n}\right\}$, where $v_{i}$ represents the block $B_{i}$ for $i \in[n]$. For any $i, j \in[n]$ and $i \neq j$, there are $d(i \rightarrow j)$ directed edges from vertex $v_{i}$ to $v_{j}$. This graph $G_{m}=(V, E)$ is called the "movement graph."

Definition 16. (Permuted LABELling, AsCending EDGES, AND DESCENDING EDGES) Let $\pi$ be a permutation of $\{1,2, \cdots, n\}$. That is, $\pi(i) \in[n]$ for any $i \in[n]$, and $\pi(i) \neq \pi(j)$ for any $i \neq j \in[n]$. Let $\Pi$ denote the set of all the $n$ ! such permutations. Let $\pi^{-1}$ be the inverse function of $\pi$.

Given a permutation $\pi$, in the movement graph $G_{m}=$ $(V, E)$, we call an edge from $v_{i}$ to $v_{j}$ an "ascending edge" if $\pi^{-1}(i)<\pi^{-1}(j)$; we call it a "descending edge" if $\pi^{-1}(i)>$ $\pi^{-1}(j)$. Let $A_{\pi}$ denote the set of ascending edges and $D_{\pi}$ denote the set of descending edges, given the permutation $\pi$. Note that $E=A_{\pi} \cup D_{\pi}$. The permutation $\pi$ is also called a "permuted labelling" of the graph $G_{m}$.

We first present a (tight) lower bound for the number of erasures. The concept of "canonical data-movement solution" used in the following proof will also be used later for proving NP hardness and approximation results.
Theorem 17. For data-movement solutions without coding, the number of erasures needed for moving data is at least

$$
n+\left\lceil\frac{\min _{\pi \in \Pi}\left|A_{\pi}\right|+\sum_{i \in[n]} d(i \rightarrow i)}{m}\right\rceil .
$$

And when $\Delta \geqslant\left\lceil\frac{\min _{\pi \in \Pi}\left|A_{\pi}\right|+\sum_{i \in[n]} d(i \rightarrow i)}{m}\right\rceil$, this bound is tight.

Proof: We present the sketch of the proof here. For details, please see [7]. Define the canonical solution to be a data-movement solution as follows: (1) Choose a permuted labelling $\pi$; (2) For $1 \leqslant i \leqslant n$ and $1 \leqslant j \leqslant m$, if $\pi^{-1}(\alpha(i, j)) \geqslant \pi^{-1}(i)$, copy the data $d_{i, j}$ to some empty page in the auxiliary blocks; (3) for $i=1,2, \ldots, n$, erase $B_{\pi(i)}$ and then write into it the data that need to be moved into it; (4) Erase all the auxiliary blocks. The canonical solution uses $n+\left\lceil\frac{\left|A_{\pi}\right|+\sum_{i \in[n]} d(i \rightarrow i)}{m}\right\rceil$ erasures and requires $\left\lceil\frac{\left|A_{\pi}\right|+\sum_{i \in[n]} d(i \rightarrow i)}{m}\right\rceil$ auxiliary blocks. Conversely, when $\Delta$ is sufficiently large, for every solution there is a corresponding canonical solution that uses no more erasures.

Theorem 18. For the generalized data-movement problem, it is NP hard to find per-instance optimal solutions without coding.

Proof: We present the sketch of the proof here. For details, please see [7]. We prove the problem is hard when $\Delta \geqslant n m$, where a canonical solution exists. Finding the optimal canonical solution is the same as find a permuted labelling $\pi$ that minimizes $\left\lceil\frac{\left|A_{\pi}\right|+\sum_{i \in[n]} d(i \rightarrow i)}{m}\right\rceil$. It is APX hard to find a permuted labelling $\pi^{*}$ that minimizes $\left|A_{\pi^{*}}\right|$, the number of ascending edges in $G_{m}$, because it is equivalent to finding the minimum set of feedback arcs in $G_{m}$. Using the approximation gap of APX, we can show that finding $\pi$ is also hard.

We can show that when $\Delta \geqslant n m$, if $c$ denotes the best approximation ratio known for the minimum arc-set problem, then we can derive a canonical solution for data movement correspondingly, which can achieve an approximation ratio of

$$
\frac{3 c}{2 c+1}+\frac{1}{n} \approx \frac{3 c}{2 c+1}<\min \left\{\frac{3}{2}, c\right\}
$$

for the number of erasures. The details are presented in [7].

\section{CONCLUSIONS}

In this paper, we present both coding and non-coding based algorithms for efficient data movement in flash memories. By proving a lower bound for the number of erasures used by algorithms without coding, we rigorously show the advantage of coding. The hardness and the approximation of per-instance optimization are also studied.

\section{APPENDIX}

We describe here an algorithm for the non-coding data movement problem that is efficient and has a straightforward implementation. The model we use is slightly different from the one in previous sections, but only for the sake of a clear description. More specifically, we model the data by an $m \times n$ matrix. Each entry is labeled with a number from 0 to $n-1$, specifying the destination block. For each label $i$, there are 
exactly $m$ entries labeled with $i$. In the construction below we use $n=m=2^{p}$ and $\Delta=2$. It is not hard to verify that the construction extends to genearal $\Delta$ and arbitrary $m \geqslant n$ (by changing the underlying alphabet used in the construction from 2 to $\Delta$ symbols).

\section{A. Decomposition by Hall's Theorem}

We can apply the well known Hall's theorem from combinatorics [2] to decompose the matrix into $n$ sets $S_{0}, \ldots, S_{n-1}$, such that each set contains all the numbers from 0 to $n-1$ exactly once, and also each set contains exactly one number from each column.

In our case a set is a column, and the family of sets is the entire matrix. It is easy to verify that the union of any $k$ columns, for all $k$ from 1 to $n$ contains at least $k$ different numbers. Therefore the theorem applies, and the decomposition can be found in $O\left(n^{3} \log n\right)$, or $O\left(m n^{2} \log n\right)$ in general [1], while this does not involve any erasure.

\section{B. Data Movement by Twice Transpose}

The data movement problem can be solved by two applications of an algorithm that realizes the transpose of the matrix. First, we decompose the matrix by Hall's theorem, to obtain the sets $S_{0}, \ldots, S_{n-1}$ as described before. With $n$ erasures, by changing the position of pages within each column, each set $S_{i}$ can be moved to occupy the $i$-th row of the matrix. After the first transpose, each set $S_{i}$ will ocuppy the $i$-th column. With $n$ more erasures, again rearrangements within each column, every set $S_{i}$ can be ordered from 0 to $n-1$. Finally, the second transpose gives the desired configuration. (An example for a $4 \times 4$ matrix is shown in [7].) The basic step of the algorithm is an exchange of entries between two columns. Therefore, the vertical position of pages in a column is not important at the beginning of the algorithm, and we can exclude the $n$ erasures that were mentioned before each transpose.

\section{Bit-Fixing Algorithm for Transpose}

We now describe the algorithm that realizes the transpose of a square matrix with $n=2^{p}$ columns. It is shown as Algorithm 19 below. The guiding principle of the algorithm is to use the binary representation of the column indexes (and of the matrix entries), and move the data between columns such that corresponding bits of the entries are in agreement with those of the columns, therefore by "fixing the bits". The outer loop has $p$ steps, corresponding to each of the $p$ bits in the binary representation. They can be fixed in any order, but for the sake of a definition we chose the one from least to most significant. For each bit that is fixed, we make $n / 2=2^{p-1}$ pairs of columns. The condition is that for each pair, the binary representation of their indexes has to agree on the bits that have already been fixed. Again, for the simplicity of defining the algorithm, we choose the pairs such that the binary representations of their indexes agree on all the bits, except the one being fixed in the current round. A pair is defined by the columns $B_{0}$ and $B_{1}$ in the algorithm. For any such pair, we rearrange their entries such that they agree with the column index on the bit $i$ that is currently being fixed.
Algorithm 19. Bit-Fixing Algorithm FOR Transpose
InPUt:
$\left(\begin{array}{cccc}0 & 0 & \text { Square } & \multicolumn{2}{c}{\text { matrix }} & 0 \\ 1 & 1 & \cdots & 1 \\ \vdots & \vdots & \cdots & \vdots \\ n-1 & n-1 & \cdots & n-1\end{array}\right)$, where $n$ is a power of $2, n=2^{p}$.

OutPut: The transpose of $\mathcal{A}$.

ALGORITHM: For $i=0$ to $p-1$, and for $k=0$ to $2^{p-1}-1$, do:

1) Let $\left(b_{p-2} \ldots b_{1} b_{0}\right)$ be the binary representation of $k$.

2) Let $B_{0}$ be the column with binary index $\left(b_{p-2} \ldots b_{i} \mathbf{0} b_{i-1} \ldots b_{1} b_{0}\right)$.

3) Let $B_{1}$ be the column with binary index $\left(b_{p-2} \ldots b_{i} \mathbf{1} b_{i-1} \ldots b_{1} b_{0}\right)$.

4) Between $B_{0}$ and $B_{1}$, move entries whose $i$-th bit is 0 to $B_{0}$ and those whose $i$-th bit is 1 to $B_{1}$.

Due to the space limit, we skip the proof for the following theorem. Interested readers can refer to [7] for details. An example of Algorithm 19 is also shown in [7].

Theorem 20. The BIT-FIXING TRANSPOSE Algorithm is correct, namely it realizes the transpose of the input matrix. The number of block erasures is $n \log n$, using only two extra blocks of memory.

\section{ACKNOWLEDGMENT}

This work was supported in part by the NSF CAREER Award CCF-0747415, NSF grant ECCS-0802107, ISF grant 480/08, and Caltech Lee Center for Advanced Networking.

\section{REFERENCES}

[1] N. Alon, "A simple algorithm for edge-coloring bipartite multigraphs," in Inf. Process. Lett., vol. 85, no. 6, pp. 301-302, 2003.

[2] B. Bollobás, Random Graphs. Cambridge University Press, 2001.

[3] G. Even, J. Naor, B. Schieber and M. Sudan, "Approximating minimum feedback sets and multi-cuts in directed graphs," in Proc. 4th Int. Conf. on Integer Prog. and Combinatorial Optimization, Lecture Notes in Comput. Sci. 920, Springer-Verlag, pp. 14-28, 1995.

[4] H. Finucane, Z. Liu, and M. Mitzenmacher, "Designing floating codes for expected performance," in Proc. Annual Allerton Conference, 2008.

[5] E. Gal and S. Toledo, "Algorithms and data structures for flash memories," ACM Computing Surveys, vol. 37, no. 2, pp. 138-163, 2005.

[6] A. Jiang, V. Bohossian, and J. Bruck, "Floating codes for joint information storage in write asymmetric memories," in Proc. IEEE Int. Symp. on Information Theory (ISIT), Nice, France, Jun. 2007, pp. 1166-1170.

[7] A. Jiang, M. Langberg, R. Mateescu and J. Bruck, "Data movement in flash memories," Caltech Technical Report, online: http : //www.paradise.caltech.edu/papers/etr097.pdf.

[8] A. Jiang, M. Langberg, M. Schwartz and J. Bruck, "Universal rewriting in constrained memories," in Proc. IEEE International Symposium on Information Theory (ISIT), Seoul, Korea, 2009, pp. 1219-1223.

[9] A. Jiang, H. Li and Y. Wang, "Error scrubbing codes for flash memories," Proc. Canadian Workshop on Inform. Theory (CWIT), Ottawa, Canada, May 2009, pp. 32-35.

[10] A. Jiang, R. Mateescu, M. Schwartz and J. Bruck, "Rank modulation for flash memories," in IEEE Transactions on Information Theory, vol. 55, no. 6, pp. 2659-2673, June 2009.

[11] A. Jiang, R. Mateescu, E. Yaakobi, J. Bruck, P. Siegel, A. Vardy and J. Wolf, "Storage coding for wear leveling in flash memories," in Proc. IEEE Int. Symp. on Inform. Theory (ISIT), 2009, pp. 1229-1233.

[12] A. Jiang, M. Schwartz and J. Bruck, "Error-correcting codes for rank modulation," Proc. IEEE International Symposium on Information Theory (ISIT), Toronto, Canada, July 2008, pp. 1736-1740.

[13] E. Yaakobi, A. Vardy, P. H. Siegel, and J. K. Wolf, "Multidimensional flash codes," in Proc. of the Annual Allerton Conference, 2008. 